\title{
Zodiacal Exoplanets in Time (ZEIT). VII. A Temperate Candidate Super-Earth in the Hyades Cluster
}

Vanderburg, Andrew; Mann, Andrew W.; Rizzuto, Aaron; Bieryla, Allyson; Kraus, Adam L.; Berlind, Perry; Calkins, Michael L.; Curtis, Jason L.; Douglas, Stephanie T.; Esquerdo, Gilbert A.

Total number of authors:

18

Published in:

Astrophysical Journal

Link to article, DOI:

$10.3847 / 1538-3881 /$ aac894

Publication date:

2018

Document Version

Publisher's PDF, also known as Version of record

Link back to DTU Orbit

Citation (APA):

Vanderburg, A., Mann, A. W., Rizzuto, A., Bieryla, A., Kraus, A. L., Berlind, P., Calkins, M. L., Curtis, J. L., Douglas, S. T., Esquerdo, G. A., Everett, M. E., Horch, E. P., Howell, S. B., Latham, D. W., Mayo, A. W., Quinn, S. N., Scott, N. J., \& Stefanik, R. P. (2018). Zodiacal Exoplanets in Time (ZEIT). VII. A Temperate Candidate Super-Earth in the Hyades Cluster. Astrophysical Journal, 156(2), [46]. https://doi.org/10.3847/1538$3881 /$ aac894

\section{General rights}

Copyright and moral rights for the publications made accessible in the public portal are retained by the authors and/or other copyright owners and it is a condition of accessing publications that users recognise and abide by the legal requirements associated with these rights.

- Users may download and print one copy of any publication from the public portal for the purpose of private study or research.

- You may not further distribute the material or use it for any profit-making activity or commercial gain

- You may freely distribute the URL identifying the publication in the public portal 


\title{
Zodiacal Exoplanets in Time (ZEIT). VII. A Temperate Candidate Super-Earth in the Hyades Cluster
}

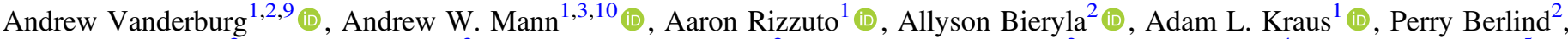 \\ Michael L. Calkins ${ }^{2}$ (1) Jason L. Curtis ${ }^{3}$ (1) Stephanie T. Douglas ${ }^{2}$ (1) , Gilbert A. Esquerdo ${ }^{2}$, Mark E. Everett ${ }^{4}$ (1), Elliott P. Horch ${ }^{5}$ (i),

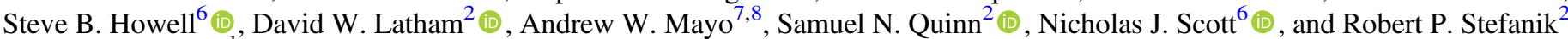 \\ ${ }^{1}$ Department of Astronomy, The University of Texas at Austin, Austin, TX 78712, USA; avanderburg@utexas.edu \\ ${ }^{2}$ Harvard-Smithsonian Center for Astrophysics, Cambridge, MA 02138, USA \\ ${ }^{3}$ Department of Astronomy, Columbia University, 550 West 120th Street, New York, NY 10027, USA \\ ${ }^{4}$ National Optical Astronomy Observatory, 950 North Cherry Avenue, Tucson, AZ 85719, USA \\ ${ }^{5}$ Department of Physics, Southern Connecticut State University, 501 Crescent Street, New Haven, CT 06515, USA \\ ${ }^{6}$ Space Science and Astrobiology Division, NASA Ames Research Center, Moffett Field, CA 94035, USA \\ ${ }^{7}$ DTU Space, National Space Institute, Technical University of Denmark, Elektrovej 327, DK-2800 Lyngby, Denmark \\ ${ }^{8}$ Centre for Star and Planet Formation, Natural History Museum of Denmark \& Niels Bohr Institute, University of Copenhagen, Øster Voldgade 5-7, DK-1350 \\ Copenhagen K., Denmark \\ Received 2018 March 11; revised 2018 May 17; accepted 2018 May 26; published 2018 July 6
}

\begin{abstract}
Transiting exoplanets in young open clusters present opportunities to study how exoplanets evolve over their lifetimes. Recently, significant progress detecting transiting planets in young open clusters has been made with the $K 2$ mission, but so far all of these transiting cluster planets orbit close to their host stars, so planet evolution can only be studied in a high-irradiation regime. Here, we report the discovery of a long-period planet candidate, called HD 283869 b, orbiting a member of the Hyades cluster. Using data from the $K 2$ mission, we detected a single transit of a super-Earth-sized $\left(1.96 \pm 0.12 R_{\oplus}\right)$ planet candidate orbiting the K-dwarf HD 283869 with a period longer than 72 days. As we only detected a single-transit event, we cannot validate HD 283869 b with high confidence, but our analysis of the $K 2$ images, archival data, and follow-up observations suggests that the source of the event is indeed a transiting planet. We estimated the candidate's orbital parameters and find that if real, it has a period $P \approx 100$ days and receives approximately Earth-like incident flux, giving the candidate a $71 \%$ chance of falling within the circumstellar habitable zone. If confirmed, HD $283869 \mathrm{~b}$ would have the longest orbital period, lowest incident flux, and brightest host star of any known transiting planet in an open cluster, making it uniquely important to future studies of how stellar irradiation affects planetary evolution.
\end{abstract}

Key words: planetary systems - planets and satellites: detection - stars: individual (HD 283869)

\section{Introduction}

The study of stars in clusters has been a cornerstone of stellar astrophysics for over a century (e.g., Russell 1914; Shapley 1917). Because clusters contain coeval stellar populations with uniform ages, compositions, and formation histories, it is possible to study stars while controlling for these variables, determine how stars of different masses appear and evolve, and understand cases where stellar evolution took unconventional paths. Stars in open clusters have enabled studies of, among other phenomena, stellar mergers (Leiner et al. 2016), mass transfer (Geller \& Mathieu 2011), rotation (Barnes 2007), and magnetic activity (Stern et al. 1981).

Now that the detection of exoplanets has gone from unproven (Struve 1952; Campbell \& Walker 1979) to achievable (Campbell et al. 1988; Latham et al. 1989; Mayor \& Queloz 1995; Butler et al. 1997; Cochran et al. 1997), to routine (Rowe et al. 2014; Morton et al. 2016; Mayo et al. 2018) over the last few decades, fundamental questions about the formation and evolution of exoplanets are becoming pertinent. Since the very first discoveries, exoplanets have been found with orbits (Mayor \& Queloz 1995; Cochran et al. 1997; Naef et al. 2001) and interior structures/ compositions (Charbonneau et al. 2009; Masuda 2014) different from those of our own solar system planets, in tension with

\footnotetext{
NASA Sagan Fellow.

${ }^{10}$ NASA Hubble Fellow.
}

traditional planet formation theories (e.g., Boss 1995). As the number of detected exoplanets grows, increasingly sophisticated analyses are beginning to yield insights into these surprising features of the exoplanet population (e.g., Dawson et al. 2015; Rogers 2015).

As astronomers begin to tackle fundamental questions about the origin and evolution of exoplanets, it stands to reason that the study of exoplanets in clusters may be similarly foundational to the study of stars in clusters. Studying a coeval planet population within a cluster could isolate trends in planet properties as a function of stellar mass (Cochran et al. 2002), while comparisons between different clusters and field populations could reveal how planet demographics depend on birth environment and how they change over time (Meibom et al. 2013; Mann et al. 2016a).

Recently, significant progress has been made detecting exoplanets in clusters. Some of the earliest discoveries came from radial velocity (RV) searches of cluster members (Lovis \& Mayor 2007; Sato et al. 2007; Quinn et al. 2012), which were generally only sensitive to giant planets. Searches for transits were originally unfruitful (Gilliland et al. 2000; Burke et al. 2006; Pepper et al. 2008) ${ }^{11}$ but found success after the launch of the Kepler space telescope, which detected two subNeptunes in the billion-year-old NCG 6811 cluster during its

\footnotetext{
${ }^{11}$ The lack of detections from transit surveys of clusters was not entirely expected (see, e.g., van Saders \& Gaudi 2011; Masuda \& Winn 2017).
} 

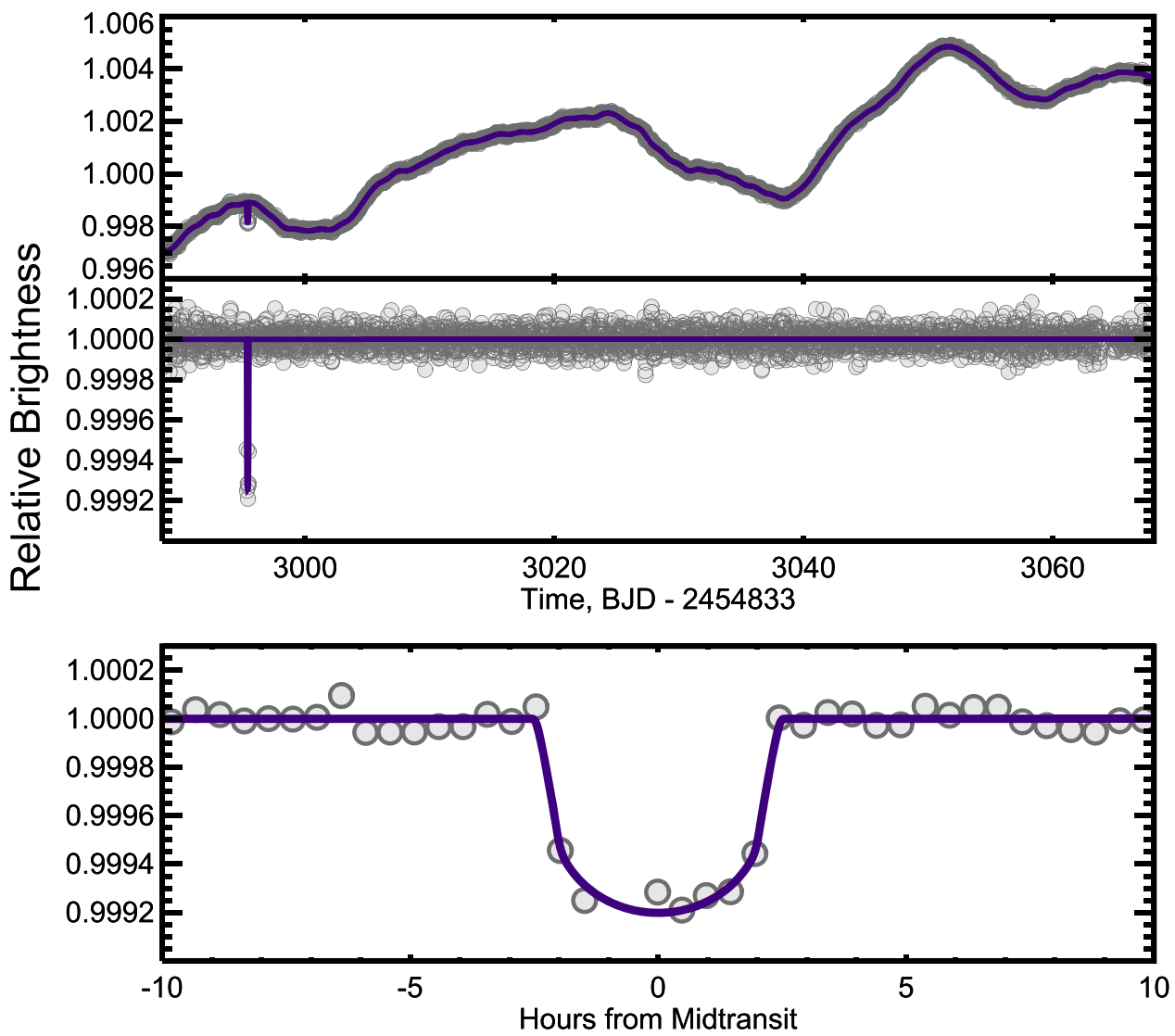

Figure 1. Top panel: systematics-corrected $K 2$ light curve of HD 283869. Gray circular points are the individual $K 2$ long-cadence flux measurements and the purple curve is the best-fit low-frequency variability and transit model. The star shows variability with a period of about 37 days-significantly longer than most other Hyades members of this mass - and a single-transit event at time BJD - $2454833 \approx 2995$. Middle panel: $K 2$ light curve with stellar variability removed. The transit signal is clearly visible, significant at the $\approx 20 \sigma$ level. Bottom panel: zoom-in of the flattened $K 2$ light curve with best-fit transit model overlaid. The signal is consistent with the transit of a super-Earth-sized exoplanet with an orbital period of about 100 days.

original mission (Meibom et al. 2013). The turning point for detecting planets in clusters came when the failure of a second reaction wheel ended the original Kepler mission and forced the spacecraft to point toward the ecliptic plane to maintain stable pointing (Howell et al. 2014). Fortuitously, a wealth of nearby and well-studied clusters and associations, including the Hyades, Praesepe, Pleiades, M67, Ruprecht 147, and Upper Scorpius, happen to lie near the ecliptic plane, making Kepler's extended $K 2$ mission well suited for detecting small transiting planets around these well-characterized stars. $K 2$ has fulfilled that promise with the detection of four planets in the Hyades (David et al. 2016a; Mann et al. 2016a, 2018; Ciardi et al. 2018; Livingston et al. 2018), six planets and one candidate in Praesepe (Libralato et al. 2016; Obermeier et al. 2016; Mann et al. 2017), one planet in Upper Scorpius (David et al. 2016b; Mann et al. 2016b), one planet in the Cas Tau association (David et al. 2018), and one planet in Ruprecht 147 (Curtis et al. 2018).

The sample of small transiting planets in open clusters is already showing intriguing patterns, perhaps hinting that planets in young clusters may be less dense than their older counterparts (Mann et al. 2016a, 2017; Obermeier et al. 2016). However, the inferences that might be made about the existing population of planets in open clusters are limited by the sample. Because of its short observing baseline, $K 2$ is most sensitive to planets in periods less than about 40 days, so the known small transiting cluster planets tend to orbit close to their host stars and be highly irradiated. Meanwhile, although RV surveys have detected some long-period, cool planets, these objects are quite massive. Currently, there are no known small planets in temperate orbits around stars in open clusters, making it impossible to study the evolution and properties of planets in low-irradiation regimes.

Here, we report the detection of a long-period transiting planet candidate around the bright $(V=10.6, K=7.7, K p=$ 10.1) Hyades member HD 283869. We detected a singletransit event in $K 2$ Campaign 13 observations of HD 283869, with a depth, duration, and shape corresponding to a superEarth in a roughly 100-day orbit around a K-dwarf stellar host. If the candidate is eventually confirmed to be real, it would be the first known temperate small planet in an open cluster. Our paper is organized as follows: In Section 2, we describe the $K 2$ discovery observations and both archival and follow-up data on HD 283869. Though we do not validate that the candidate is indeed an exoplanet with high confidence, our analysis of $K 2$ data, spectroscopy, and imaging suggests this is likely the case. In Section 3, we perform an analysis to determine stellar and planetary parameters under the assumption that the single-transit event we see is indeed due to an exoplanet. In Section 4, we discuss the uniqueness of the candidate around HD 283869 and explore the path toward confirming the transits to enable further study. 

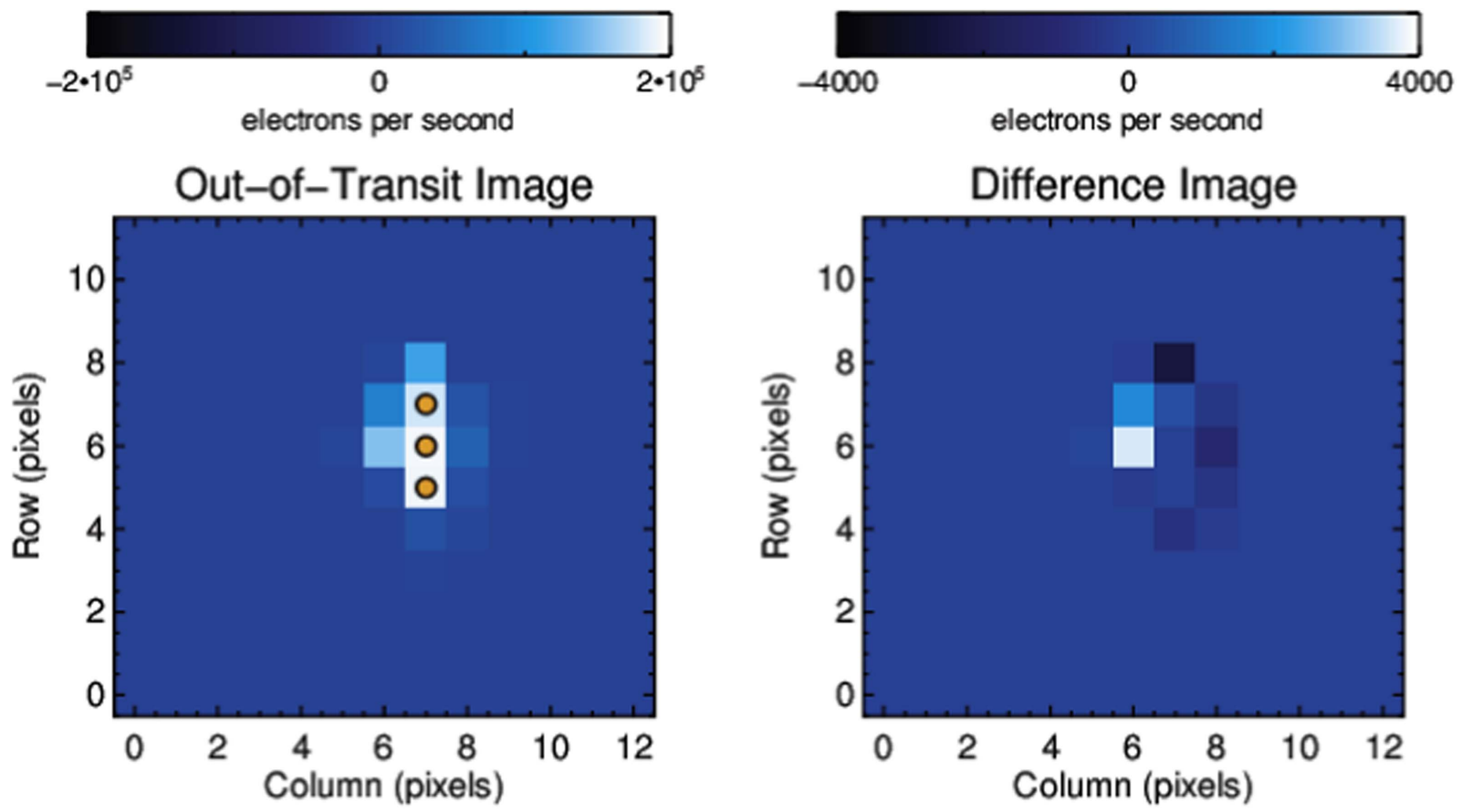

Figure 2. Difference image analysis for the candidate transit event around HD 283869. Left panel: out-of-transit image taken shortly after transit when Kepler was at nearly the same position in its roll as during the middle of the candidate transit event. The orange dots mark three saturated pixels (with electron fluxes greater than about $1.6 \times 10^{5} \mathrm{~s}^{-1}$, van Cleve \& Caldwell 2016). Right panel: difference image calculated by subtracting a $K 2$ image taken during the transit from the out-of-transit image. While difference images for saturated stars observed by Kepler are tricky to interpret, the source of the transit is on target. The morphology of the difference image is consistent with a genuine transit of HD 283869.

\section{Observations}

\subsection{K2 Light Curve}

Kepler observed part of the Hyades cluster, including HD 283869, designated EPIC 248045685, during the 13th campaign of its extended $K 2$ mission between 2017 March 8 and May 27. After the data were downlinked from the spacecraft, they were processed by the $K 2$ mission pipeline and released to the public. We downloaded the calibrated target pixel files from the Mikulski Archive for Space Telescopes, produced light curves, and removed systematic errors caused by Kepler's unstable pointing using the method described by Vanderburg \& Johnson (2014). We searched the processed light curves for transits using a Box-Least-Squares algorithm (Kovács et al. 2002; Vanderburg et al. 2016b). Even though our transit search algorithm is designed to identify periodic phenomena, it detected a single, high signal-to-noise ${ }^{12}$ transit-like dip in the brightness of HD 283869. The dip had a depth of about $800 \mathrm{ppm}$, a duration of about $4.6 \mathrm{hr}$, and a shape characterized by a rounded bottom and short ingress and egress times, consistent with the transit of a small exoplanet.

Upon identifying the transit-like event, we re-processed the $K 2$ light curve by fitting a systematics model simultaneously with the long-timescale variability of the star and a single transit of a long-period planet (see Vanderburg et al. 2016b, for details). Our final $K 2$ light curve is shown in Figure 1. The $K 2$ light curve is dominated by a long-period signal, which we think is likely astrophysical and could be related to stellar rotation. We measured a period of about $37 \pm 2$ days in the $K 2$ light curve using both an autocorrelation function and

$\overline{12}$ We estimate the signal-to-noise of the dip is roughly 21 .
Lomb-Scargle analysis. If this period is in fact the rotation period of the star, then HD 283869 is an anomalously slow rotator for a star of its mass and age; most single Hyades and Praesepe members with similar masses have rotation periods of about 10-15 days. We discuss this point further in Section 4. When the long-period signal is removed, the dip is clearly visible by eye near the beginning of the $K 2$ light curve.

While $K 2$ data are typically quite reliable, occasionally single events like the one we detect in the light curve of HD 283869 can be caused by instrumental phenomena. We therefore subjected the single dip to a battery of tests to rule out various scenarios that we have observed to cause similar signals in $K 2$ data in the past. In particular, we confirmed that there were no changes to the scattered background light (perhaps caused by a bright solar system object moving across Kepler's focal plane ${ }^{13}$ ) during the $4.6 \mathrm{hr}$ transit-like event. We also confirmed that the dip was not a residual of our correction for systematics caused by $K 2$ 's repeated drifting motion and thruster corrections. The dip spanned two drift periods and took place while Kepler was oriented in a part of its roll that was well-characterized by our "self flat field" systematics correction. We also inspected the light curves of the two other stars ${ }^{14}$ observed by $K 2$ within 5 arcmin of HD 283869 and found no similar simultaneous dips, indicating that the transitlike-event was not caused by some wide-reaching detector anomaly. We performed standard $K 2$ pixel-level tests (see,

\footnotetext{
${ }^{13}$ For an example of such a scenario, see Figure 4(b) of Vanderburg (2014), which shows a spurious single transit-like event caused by an increase in scattered background light as the planet Jupiter moved out of Kepler's focal plane.

${ }^{14}$ In particular, https://archive.stsci.edu/prepds/k2sff/html/c13/ep248053336. html and https://archive.stsci.edu/prepds/k2sff/html/c13/ep248053424.html.
} 
POSS I Blue: 1955

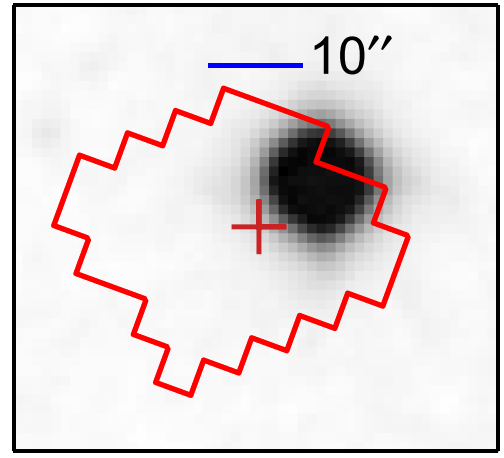

POSS II Red: 1991

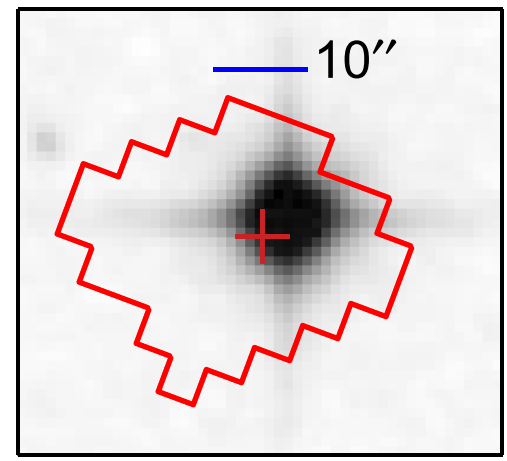

K2: 2017

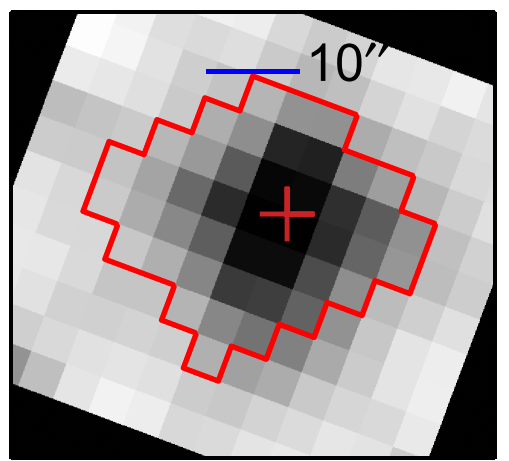

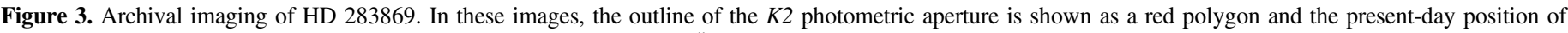

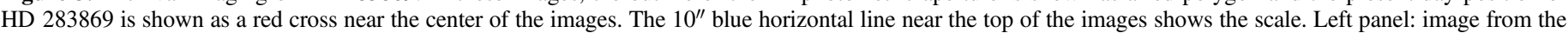

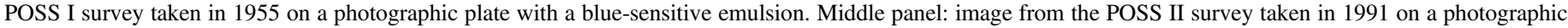

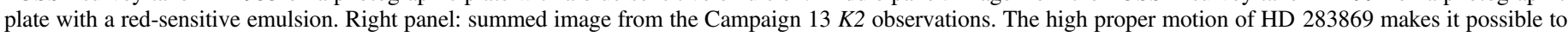
exclude bright background companions at the star's present-day location.

e.g., Vanderburg et al. 2016b; Mayo et al. 2018) and confirmed that the apparent position of the star did not shift appreciably during the transit-like event both by difference image analysis (see Figure 2) and analysis of measured image centroids. ${ }^{15}$ Finally, we showed that the shape and depth of the transit remained the same when the photometric aperture used to extract the light curve was changed.

Based on these tests, we conclude that the transit-like event we see is probably caused by some astrophysical phenomenon in the direction of HD 283869, and throughout the rest of the paper, we proceed under this assumption. In Sections 2.2 and 2.3, we go further and argue that that the most likely explanation for the dip in the light curve of HD 283869 is that the star is indeed transited by a small, long-period exoplanet, but we do not go so far as to attempt to validate the signal as being caused by a genuine exoplanet with high confidence. Instead, given the difficulty of ruling out all possible false positive scenarios for single-transit events, we consider the likely source of the signal to be a "planet candidate," which it will remain until it is confirmed by the detection of additional transits or through precise Doppler monitoring (e.g., Vanderburg et al. 2015). For convenience, throughout the rest of the paper, we refer to the planet candidate as HD $283869 \mathrm{~b}$.

\subsection{Spectroscopy}

HD 283869 is a well studied star thanks to its long-suspected Hyades membership. Here, we make use of extensive archival observations and some new observations taken after we identified the planet candidate orbiting HD 283869.

After being identified as a candidate Hyades member by photometric and proper motion surveys, HD 283869 was observed spectroscopically three times between 1974 and 1980 with the Radial Velocity Spectrometer at the Coudé focus of the $5.1 \mathrm{~m}$ Palomar Hale telescope (Griffin et al. 1988) as part of a survey to identify true Hyades members among previously

\footnotetext{
${ }^{15}$ With a Kepler-band magnitude of 10.15 , the image of HD 283869 is saturated in the $K 2$ images, which can confuse diagnostics like image centroid shifts and difference images. Nevertheless, with the difference image analysis, we are able to show that the source of the transit is cospatial with HD 283869 , and we are able to confirm that the shift in image centroids (transverse to the spacecraft roll) during transit is less than about 2 mas compared to the spacecraft position in the two days surrounding the transit.
}

identified candidates. The three RV measurements from this survey had a mean velocity of $39.6 \pm 0.17 \mathrm{~km} \mathrm{~s}^{-1}$ on the IAU system $^{16}$ (with no variations at the $500 \mathrm{~m} \mathrm{~s}^{-1}$ level), suggesting kinematics consistent with Hyades membership. ${ }^{17}$

Some of us began observing HD 283869 in 1991 as part of an RV survey of Hyades members using the CfA Digital Speedometers on the $1.5 \mathrm{~m}$ Wyeth Reflector at Oak Ridge Observatory in the town of Harvard, MA and on the $1.5 \mathrm{~m}$ Tillinghast Reflector at Fred L. Whipple Observatory on Mt. Hopkins, AZ (Stefanik et al. 1985). We obtained a total of 17 observations with the CfA Digital Speedometers between 1991 and 2006, all but two of which came from Oak Ridge Observatory. The RV time series shows no convincing evidence for astrophysical variability at the $300 \mathrm{~m} \mathrm{~s}^{-1}$ level, and a periodogram search reveals no strong periodicities. The mean velocity of the 17 Digital Speedometer observations is $39.7 \pm 0.13 \mathrm{~km} \mathrm{~s}^{-1}$ on the IAU scale. There is no significant velocity difference between the CfA observations and the Palomar observations taken two decades earlier.

More recently, we observed HD 283869 with the Tillinghast Reflector Echelle Spectrograph (TRES), the high-resolution successor to the CfA Digital Speedometers on the $1.5 \mathrm{~m}$ telescope at Mt. Hopkins. We obtained one observation in 2011 October and two other observations in 2017 September after we identified the planet candidate. We measured relative radial velocities between the three TRES observations using methods developed by Buchhave et al. (2010). We detect a possible $80 \mathrm{~m} \mathrm{~s}^{-1} \mathrm{RV}$ shift between the observation taken in 2011 and the two observations taken in 2017 , but the formal confidence of this shift is only about $2 \sigma$, and we do not consider it significant. When placed on the IAU scale, the average of the three TRES RVs is $39.84 \pm 0.1 \mathrm{~km} \mathrm{~s}^{-1}$, where the uncertainty is dominated by the transfer onto the IAU system. We adopt this value for the absolute RV.

\footnotetext{
${ }^{16}$ Griffin et al. (1988) measured a mean velocity of $40.3 \mathrm{~km} \mathrm{~s}^{-1}$. We offset the Griffin et al. (1988) velocities to the IAU system by applying a correction of $-0.84 \mathrm{~km} \mathrm{~s}^{-1}$ between the Griffin et al. (1988) system and the CfA Digital Speedometer system, which we derived from observations of constant-velocity targets in common between the Griffin et al. (1988) and CfA programs. Once the velocities were on the CfA system, we shifted them to the IAU system by applying a correction of $+0.14 \mathrm{~km} \mathrm{~s}^{-1}$.

17 The mean Hyades RV is $39.3 \pm 0.25 \mathrm{~km} \mathrm{~s}^{-1}$ with a velocity dispersion is $2.8 \mathrm{~km} \mathrm{~s}^{-1}$ (Mermilliod et al. 2009).
} 


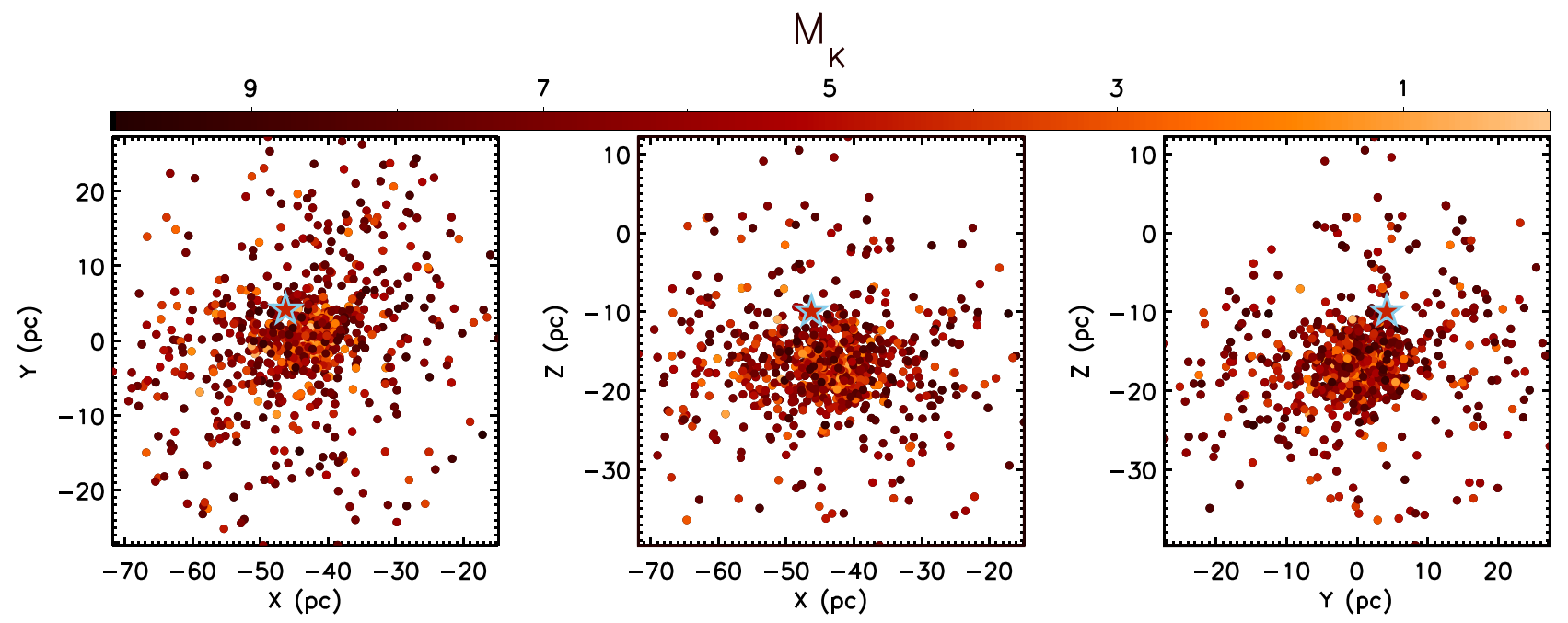

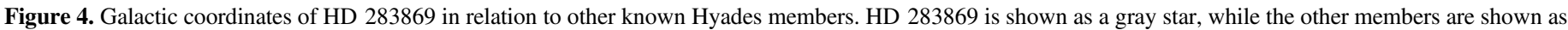

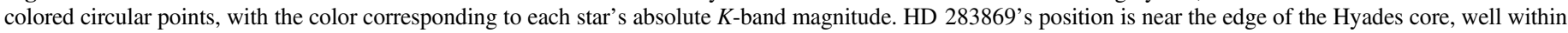
the larger distribution of Hyades members.

The most precise existing RV observations of HD 283869 were conducted as part of a survey to detect giant planets in the Hyades using the High Resolution Echelle Spectrograph (HIRES) on the $10 \mathrm{~m}$ Keck I telescope on Maunakea, HI (Cochran et al. 2002; Paulson et al. 2004). HD 283869 was observed six times between 1998 and 2003 with typical uncertainties of about $5 \mathrm{~m} \mathrm{~s}^{-1}$. We placed limits on radial acceleration on HD 283869 by fitting the six HIRES RV measurements with a linear model while allowing for an RV "jitter" term. We found no statistically significant acceleration, measuring a best-fit slope of about $3 \pm 2 \mathrm{~m} \mathrm{~s}^{-1} \mathrm{yr}^{-1}$, roughly the acceleration induced by either a Jupiter mass planet at 5 au, or a $0.1 M_{\odot}$ M-dwarf at 50 au. Significantly closer or moremassive objects than this must be nearly face-on in order to escape detection.

All in all, four decades of spectroscopic observations of HD 283869 show no evidence for RV variations, placing strong limits on the presence of binary companions. The lack of detected RV variations show definitely that HD 283869 is not a short-period eclipsing binary, eliminating that false positive scenario for the planet candidate. The RV constraints also place limits on the presence of distant companions that might be eclipsing systems themselves, decreasing the likelihood of a hierarchical eclipsing binary false positive scenario.

\subsection{Imaging}

We used a combination of archival imaging and newly acquired high angular resolution images to search for visual companions to HD 283869. We first inspected images taken in the original Palomar Observatory Sky Survey (POSS) on a photographic plate with a blue-sensitive emulsion to search for stationary background objects close to the present-day position of HD 283869. Since HD 283869 was observed by POSS in 1955, its apparent position in the sky has moved by about 9 arcsec, making it possible to search for stationary background stars near the its present-day position (see Figure 3). In a bluesensitive plate, the saturated point-spread function of HD 283869 extends near its present-day position 9 arcsec away, and we see no evidence for any elongation that might hint at a background star in the present-day location of HD 283869. We estimate based on the other nearby faint stars

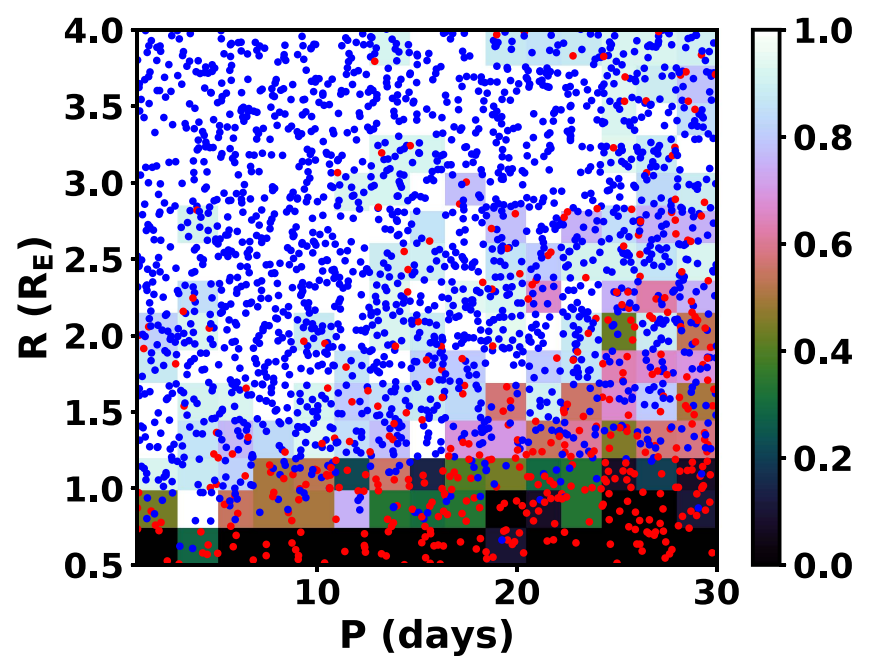

Figure 5. Sensitivity to additional transiting planets around HD 283869. We show the orbital periods and planet radii of our injected planets as circular points in the plot; blue points represent planets that we successfully recovered with our notch-filter pipeline, and red points indicate planets that we did not recover. The plot background color shows the fraction of recovered planets in each region of parameter space.

in the POSS image that if there was a star brighter than about 18th magnitude at the present-day position of HD 283869, we would have seen it. Because we see no such star close to the present-day position of HD 283869, we can exclude background stars about six magnitudes fainter in blue bandpasses. We also searched for wide co-moving binary companions using the Hot Stuff for One Year catalog (Altmann et al. 2017). We identified no other stars out to a distance of 900 arcsec (about 40000 au projected distance) brighter than $R \approx 19$ (corresponding to roughly $0.1 M_{\odot}$ M-dwarfs) with a proper motion consistent with HD 283869. Finally, we queried the Gaia DR2 database for sources near HD 283869 (Gaia Collaboration et al. 2016b, 2018). Gaia identified three very faint point sources within the $K 2$ photometric aperture at distance of 3 !! 7,9 .! 2 , and 12!"8. These point sources are too faint for Gaia to have measured proper motions or parallaxes, so we cannot ascertain 

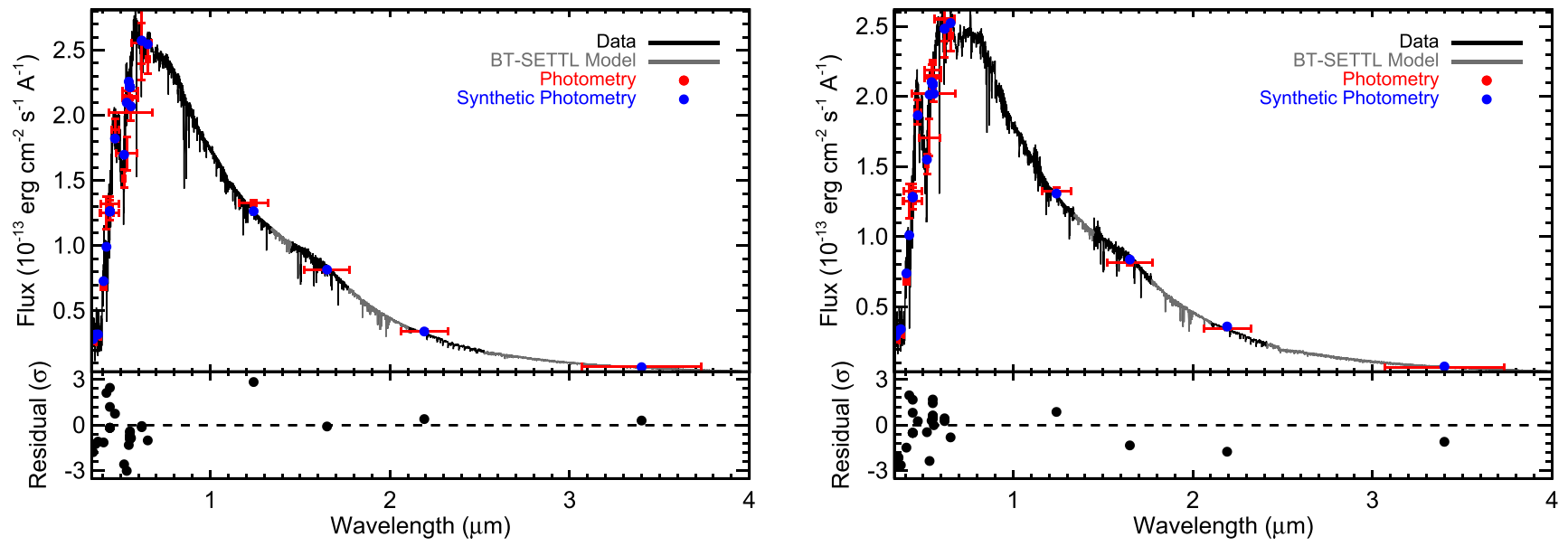

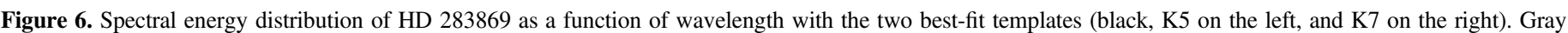

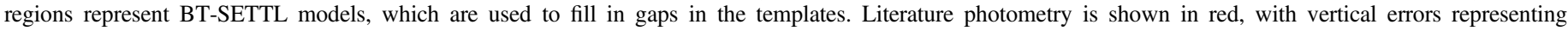

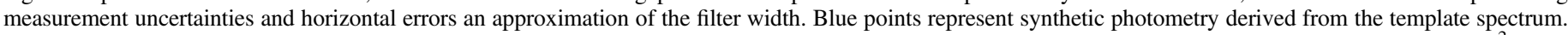

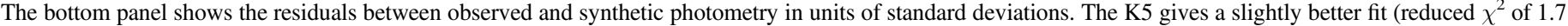
and 2.0).

whether any of them are physically associated with HD 283869 or if they are background objects. All three of these stars have Gaia-band $G$ magnitudes fainter than $G=19.4$, too faint to have caused the $700 \mathrm{ppm}$ transit signal we observed on HD 283869. Evidently, there are no widely separated stars near HD 283869 that could have contributed the transit signal we see.

After identifying the planet candidate, we observed HD 283869 with two speckle imaging instruments: the NNExplore Exoplanet Stellar Speckle Imager (NESSI) on the $3.5 \mathrm{~m}$ WIYN telescope on Kitt Peak in Arizona, and 'Alopeke on the $8 \mathrm{~m}$ Gemini-N telescope on Maunakea, HI. NESSI and 'Alopeke both work by taking many short (40-60 ms) exposures of a target star simultaneously in two optical narrow bands. The short exposures freeze out atmospheric turbulence, resulting in sub-images that can be reconstructed using Fourier techniques to produce diffraction-limited images over small fields of view. We observed with NESSI in $40 \mathrm{~nm}$ wide filters centered at 562 and $832 \mathrm{~nm}$ and with 'Alopeke in similar filters centered at 562 and $880 \mathrm{~nm} .{ }^{18}$ We reduced the data using the method described by Howell et al. (2011) and detected no nearby companions in any of the reconstructed images. The strongest constraints at small angular separations are placed by 'Alopeke; we can exclude stars 4.4 magnitudes fainter at angular separations of 0.1 arcsec (or projected distances of $5 \mathrm{au}$ ). The NESSI images are deeper than the 'Alopeke images due to observing conditions and contribute the strongest constraints at larger angular distances. The NESSI data at $832 \mathrm{~nm}$ exclude stars about 5.8 magnitudes fainter at this wavelength at distances of about $1 \mathrm{arcsec}$, or projected distances of $50 \mathrm{au}$.

The constraints we place on background objects and visual companions from archival and speckle imaging further limit false positive scenarios, making it more likely that the planet candidate around HD 283869 is indeed a transiting exoplanet. Therefore, throughout the rest of this paper, we perform analyses assuming that HD 283869 is single and that the candidate transit event is indeed caused by a transiting exoplanet.

\footnotetext{
18 Due to poor weather conditions for our observation with 'Alopeke, only the image taken with the $880 \mathrm{~nm}$ filter was usable.
}

\section{Analysis}

\subsection{Membership in the Hyades}

HD 283869 has a long history of being associated with the Hyades cluster. Griffin et al. (1988) measured an RV for HD 283869 consistent with Hyades membership, but they flagged it as a possible member, citing inconsistencies in literature proper motion measurements as a source of doubt. More recently, Perryman et al. (1998) and Röser et al. (2011) assigned HD 283869 membership using updated astrometric parameters from Hipparcos (ESA 1997) and the PPMXL catalogs, respectively.

We reassessed the case for HD 283869's membership in the Hyades. First, we note that there is solid evidence for HD 283869's membership based on its position and proper motion. HD 283869 is located near the outskirts of the Hyades core (see Figure 4), and the star's space velocity is toward the cluster's convergence point. (The star has a velocity of $23.7 \mathrm{~km} \mathrm{~s}^{-1}$ parallel to the cluster's convergence point and only $1.3 \mathrm{~km} \mathrm{~s}^{-1}$ perpendicular to the convergence point, Röser et al. 2011.) Using the methods described by Rizzuto et al. (2011) and Rizzuto et al. (2015), and the Hyades cluster model from Rizzuto et al. (2017), we calculate a membership probability greater than $99 \%$. This calculation does not take into account the measured RV (consistent with Hyades membership) and the fact that HD 283869 falls right on the Hyades main sequence in a color-magnitude diagram. Including this additional information brings the membership probability to near unity. Although HD 283869 has a slightly discrepant proper motion perpendicular to the cluster convergence point (larger than all but a handful of other known members) and might have an anomalously long rotation period (see Section 4.2), the preponderance of the evidence suggests that it is indeed a Hyades member.

\subsection{Limits on Additional Transiting Planets}

We placed limits on additional (short-period) transiting planets by performing injection/recovery tests following the procedure outlined by Rizzuto et al. (2017). We injected 4000 transit signals with randomly chosen planet and orbital 

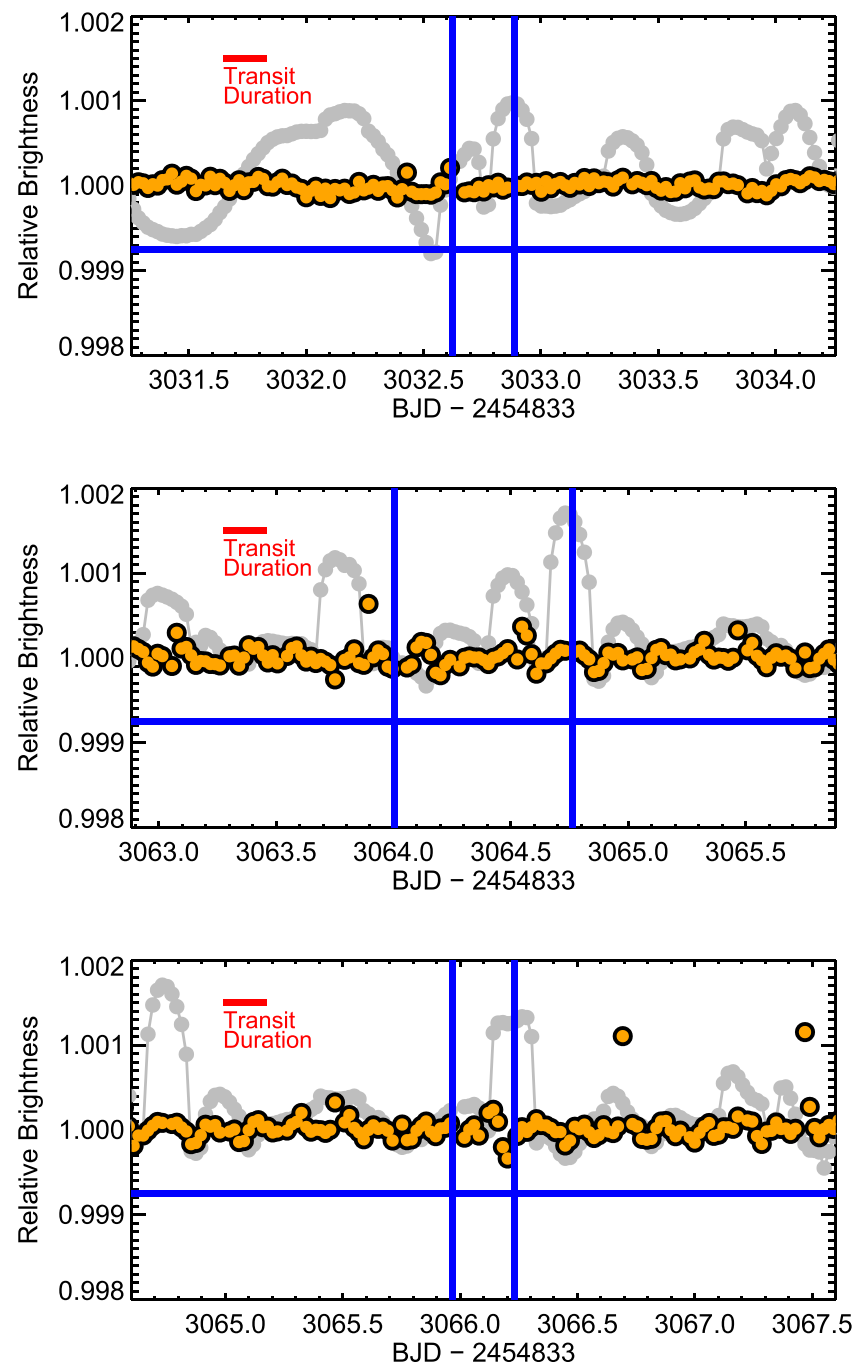

Figure 7. $K 2$ light curve during periods when the spacecraft lost fine-pointing control. Each panel shows both the systematics-corrected $K 2$ light curve (orange) and the raw $K 2$ light curve convolved with the shape of HD 283869 b's transit (gray) to partially average over the uncorrected $K 2$ roll systematics. We show the raw $K 2$ light curve in addition to the more precise systematics-corrected light curve to demonstrate that no plausible transit signals were absorbed by the systematics correction in these poorly constrained parts of the flat field. The periods when $K 2$ lost fine-pointing control are interior to the two horizontal blue lines, and the depth of HD 283869 b's transit is shown with the horizontal blue line. The duration of HD 283869 b's transit is shown as a red horizontal line in the upper left-hand corner of each panel. There are no signals in either the raw or systematicscorrected light curves during the periods without fine-pointing control consistent with a second transit of HD 283869 b.

parameters into the light curve of HD 283869 and attempted to recover them with the "notch-filter" pipeline described by Rizzuto et al. (2017). Our results are shown in Figure 5. We find that we are generally sensitive to sub-Earth-sized planets in short-period ( $\lesssim 5$ days) orbits and somewhat sensitive to Earthsized planets out to periods of about 25 days. If there are other similarly sized planets orbiting interior to HD $283869 \mathrm{~b}$, then there must be some misalignment between the planets' orbits.

\subsection{Stellar Parameters}

We used the Stellar Parameter Classification (SPC; Buchhave et al. 2012, 2014) method to determine the effective temperature, surface gravity, and equatorial rotational velocity of HD 283869 from the three TRES spectra. We ran SPC while fixing the metallicity to the cluster metallicity; we used a value of +0.15 that is an average of several previous determinations (Paulson et al. 2003; Dutra-Ferreira et al. 2016). Averaging the results for each of the three spectra, we measure a temperature $T_{\mathrm{eff}, \mathrm{SPC}}=4686 \pm$ $50 \mathrm{~K}$, surface gravity $\log g_{\mathrm{SPC}}=4.70 \pm 0.1$, and we place an upper limit on the star's projected equatorial rotation velocity of about $2 \mathrm{~km} \mathrm{~s}^{-1}$. We measure an average Mt. Wilson activity $R_{\mathrm{HK}}^{\prime}$ indicator from our three TRES spectra of $R_{\mathrm{HK}}^{\prime}=-4.77 \pm 0.05$ using the procedure described by Mayo et al. (2018).

We estimated the luminosity of HD 283869 using the parallax from Gaia DR1 (21.05 \pm 0.29 mas, Gaia Collaboration et al. $2016 \mathrm{a})^{19}$ and fitting empirical templates to the available photometry, following the procedure from Mann et al. (2015, 2017), which we briefly describe here. We first downloaded archival photometry from the literature, including $J H K_{S}$ from the Two Micron All Sky Survey (Skrutskie et al. 2006), $B_{T}$ and $V_{T}$ from Tycho-2 (Høg et al. 2000), $H_{P}$ from Hipparcos (van Leeuwen et al. 1997), $U B V$ from the General Catalogue of Photometric Data (Mermilliod et al. 1997), $B V$ and $r^{\prime}$ from the AAVSO All-Sky Photometric Survey (Henden et al. 2012), $r^{\prime}$ from the Carlsberg Meridian Catalogue (Muiños \& Evans 2014), and W1 W2 W3 W4 from the Wide-field Infrared Survey Explorer (Wright et al. 2010).

We converted literature photometry to fluxes using the appropriate filter profile and zero-point (e.g., Cohen et al. 2003; Bessell \& Murphy 2012; Mann \& von Braun 2015). Utilizing spectra from the IRTF Cool Stars Library (Cushing et al. 2005; Rayner et al. 2009) and CONCH-SHELL catalog (Gaidos et al. 2014), we found the best-fit spectral template by comparing these fluxes to values derived from these spectra, allowing the mean flux level of the template to float (Figure 6). We filled in regions of high telluric contamination and those not covered by our templates using BT-SETTL models (Allard et al. 2011). Given that the star is within the "Local Bubble," reddening is likely to be negligible (Lallement et al. 2003) and was not included in our analysis. The final bolometric flux was taken to be the integral over all wavelengths of the best-fit template and model, scaled to match the photometry. Interpolating between templates gave a negligible improvement in the fit (improvement in reduced $\chi^{2}$ of $<0.1$ ). Uncertainty on the bolometric flux was calculated by accounting for errors in the individual magnitudes, zero-points, and differences between templates. This procedure yielded a bolometric flux of $2.61 \pm 0.05 \times 10^{-9} \mathrm{erg} \mathrm{cm}^{-2} \mathrm{~s}^{-1}$. Combined with the Gaia DR1 parallax (21.05 \pm 0.29 mas), this gave a luminosity of $0.182 \pm 0.006 L_{\odot}$.

To determine other stellar parameters, we interpolated this luminosity onto the Mesa Isochrones and Stellar Tracks (Choi et al. 2016; Dotter 2016) and Dartmouth Stellar Evolution Program (Dotter et al. 2008), using the canonical Hyades age (600-800 Myr Perryman et al. 1998; Brandt \& Huang 2015; Martín et al. 2018) and metallicity ( $\simeq 0.15$, Liu et al. 2016). Accounting for differences between the two model grids, and errors on the input parameters, this procedure gives $T_{\text {eff }}=4655 \pm 55 \mathrm{~K}, \quad R_{*}=0.664 \pm 0.023 M_{\odot}, \quad$ and $\quad M_{*}=$ $0.742 \pm 0.023 M_{\odot}$. This $T_{\text {eff }}$ is consistent with the value derived from the TRES spectrum. We also obtained a

\footnotetext{
19 Recently, a more precise parallax for HD 283869 was included in Gaia DR2 of $21.003 \pm 0.054$ mas. We confirmed that the stellar parameters and uncertainties derived using this new parallax remain consistent within errors, and the uncertainties in stellar parameters, which are dominated by systematic errors in stellar evolutionary models, were unchanged.
} 
Table 1

System Parameters for HD 283869

\begin{tabular}{|c|c|c|c|c|}
\hline Parameter & Value & & $\begin{array}{l}\text { 68.3\% Confidence } \\
\text { Interval Width }\end{array}$ & Comment \\
\hline \multicolumn{5}{|l|}{ Other Designations } \\
\hline \multicolumn{5}{|l|}{ EPIC 248045685} \\
\hline \multicolumn{5}{|l|}{ HIP 22271} \\
\hline \multicolumn{5}{|l|}{$\mathrm{BD}+25733$} \\
\hline \multicolumn{5}{|l|}{ Basic Information } \\
\hline R.A. & 04:47:41.80 & & & A \\
\hline Decl. & $+26: 09: 00.8$ & & & A \\
\hline Proper Motion in R.A. [mas $\mathrm{yr}^{-1}$ ] & 113.42 & \pm & 0.18 & A \\
\hline Proper Motion in decl. [mas $\mathrm{yr}^{-1}$ ] & -83.83 & \pm & 0.12 & $\mathrm{~A}$ \\
\hline Absolute Radial Velocity $\left[\mathrm{km} \mathrm{s}^{-1}\right]$ & 39.84 & \pm & 0.1 & $\mathrm{~B}$ \\
\hline Distance to Star $[\mathrm{pc}]$ & 47.51 & \pm & 0.65 & A \\
\hline$V$-magnitude & 10.60 & \pm & 0.012 & $\mathrm{~A}$ \\
\hline$K$-magnitude & 7.72 & \pm & 0.03 & A \\
\hline Kepler-band $K p$ magnitude & 10.15 & & & $\mathrm{~A}$ \\
\hline Mt. Wilson $R_{\mathrm{HK}}^{\prime}$ & -4.77 & \pm & 0.05 & $\mathrm{~B}$ \\
\hline \multicolumn{5}{|l|}{ Stellar Parameters } \\
\hline Mass $M_{\star}\left[M_{\odot}\right]$ & 0.74 & \pm & 0.03 & $\mathrm{C}$ \\
\hline Radius $R_{\star}\left[R_{\odot}\right]$ & 0.66 & \pm & 0.03 & $\mathrm{C}$ \\
\hline Luminosity $L_{\star}\left[L_{\odot}\right]$ & 0.182 & \pm & 0.006 & $\mathrm{C}$ \\
\hline Limb-darkening $u_{1}$ & 0.570 & \pm & 0.062 & $\mathrm{D}, \mathrm{E}$ \\
\hline Limb-darkening $u_{2}$ & 0.043 & \pm & 0.068 & $\mathrm{D}, \mathrm{E}$ \\
\hline $\log g_{\mathrm{SPC}}[\mathrm{cgs}]$ & 4.7 & \pm & 0.1 & $\mathrm{~B}$ \\
\hline Metallicity $[\mathrm{M} / \mathrm{H}]$ & 0.15 & \pm & 0.03 & $\mathrm{~F}$ \\
\hline$T_{\text {eff }}[\mathrm{K}]$ & 4655 & \pm & 55 & $\mathrm{C}$ \\
\hline$v \sin i\left[\mathrm{~km} \mathrm{~s}^{-1}\right]$ & $<2$ & & & $\mathrm{~B}$ \\
\hline \multicolumn{5}{|l|}{$H D 283869 b$} \\
\hline Orbital Period, $P$ [days] & 106 & & ${ }_{-25}^{+74}$ & $\mathrm{C}, \mathrm{D}$ \\
\hline Radius Ratio, $R_{P} / R_{\star}$ & 0.0272 & \pm & 0.0012 & $\mathrm{C}, \mathrm{D}$ \\
\hline Scaled semimajor axis, $a / R_{\star}$ & 129 & & ${ }_{-22}^{+55}$ & C, D \\
\hline Orbital inclination, $i$ [deg] & 89.744 & & ${ }_{-0.085}^{+0.131}$ & C, D \\
\hline Transit impact parameter, $b$ & 0.64 & & ${ }_{-0.31}^{+0.13}$ & $\mathrm{C}, \mathrm{D}$ \\
\hline Transit Duration, $t_{14}[\mathrm{hr}]$ & 4.600 & \pm & 0.097 & $\mathrm{D}$ \\
\hline Time of Transit $t_{t}$ [BJD] & 2457828.3869 & \pm & 0.0011 & $\mathrm{D}$ \\
\hline Planet Radius $R_{P}\left[R_{\oplus}\right]$ & 1.96 & \pm & 0.13 & $\mathrm{C}, \mathrm{D}$ \\
\hline$T_{\mathrm{eq}}=T_{\mathrm{eff}}(1-\alpha)^{1 / 4} \sqrt{\frac{R_{\star}}{2 a}}[\mathrm{~K}]$ & 255 & & ${ }_{-44}^{+38}$ & $\mathrm{~B}, \mathrm{C}, \mathrm{D}, \mathrm{G}$ \\
\hline
\end{tabular}

Note. A: Parameters come from the EPIC catalog (Huber et al. 2016) and Gaia Data Release 1 (Gaia Collaboration et al. 2016a). B: Parameters come from analysis of the three TRES spectra. C: Parameters come from measuring the bolometric flux and luminosity using archival photometry and the Gaia parallax, and interpolating the measured stellar luminosity onto Hyades-age isochrones as described (Section 3.3). D: Parameters come from analysis of the K2 light curve (Section 3.4) with priors on the orbital period imposed (Section 3.5). E: Gaussian priors of imposed on $u_{1}$ and $u_{2}$ centered on 0.644 and 0.096, respectively, with width 0.07. F: The stellar metallicity is assumed to be the cluster metallicity. G: The equilibrium temperature $T_{\mathrm{eq}}$ is calculated assuming albedo $\alpha$ uniformly distributed between 0 and 0.7 and perfect heat redistribution.

consistent radius using the Stefan-Boltzmann relation with the TRES $T_{\text {eff }}$ and above luminosity, and a consistent mass using the empirical mass-luminosity relation from Henry \& McCarthy (1993), suggesting that the model-derived parameters are reasonable for this star.

\subsection{Transit Light Curve}

We determined transit parameters by fitting the $K 2$ light curve with a Mandel \& Agol (2002) model $^{20}$ using a Markov Chain Monte Carlo (MCMC) algorithm with affine invariant ensemble sampling (Goodman \& Weare 2010). Often, when astronomers fit transits, they parameterize planetary orbits with physical variables such as the orbital inclination $i$ or the ratio of

\footnotetext{
${ }^{20}$ We accounted for the 29.4-minute Kepler long-cadence integration time by oversampling the model light curve by factor of 30 and performing a trapezoidal integration.
}

the planet's semimajor axis to the stellar radius $a / R_{\star}$. The large uncertainties and covariances in the orbital elements of singly transiting planets make it difficult for MCMC explorations to converge in situations like that of HD 283869. Therefore, instead of using a physical parameterization, we fit the $K 2$ light curve in terms of variables directly related to the shape of the transit. In particular, we fit the transit in terms of the planet-star radius ratio, $R_{p} / R_{\star}$, the full duration of the transit from first to fourth contact, $t_{14}$, the time of transit center $t_{t}$, the transit impact parameter, $b$, and linear and quadratic limb-darkening coefficients, $u_{1}$ and $u_{2}$. We also fit for a "jitter" term describing the uncertainty in the flux in each $K 2$ long-cadence data point. We imposed priors requiring both the transit duration and the flux uncertainty term to be greater than zero and requiring the impact parameter to be between 0 and $1+R_{p} / R_{\star}$. We imposed informative Gaussian priors on $u_{1}$ and $u_{2}$, centered on the values interpolated from limb-darkening models (0.644 and 


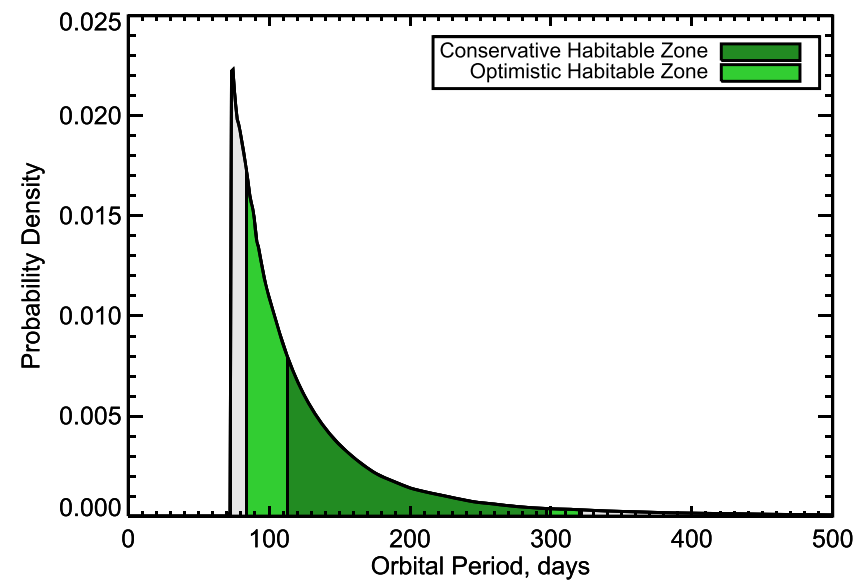

Figure 8. Constraints on the orbital period of HD 283869 b. The black curve shows the probability distribution of the orbital period from our analysis in Section 3.5. The light green and dark green shaded regions represent orbits that fall in the optimistic and conservative circumstellar habitable zones, respectively (Kopparapu et al. 2013). Despite our weak constraint on orbital period, we can say fairly confidently that if real, HD 283869 b is temperate, with a $71 \%$ chance of orbiting within the star's habitable zone and a $99 \%$ upper limit on equilibrium temperature of $327 \mathrm{~K}$.

0.096 for $u_{1}$ and $u_{2}$, respectively, Claret \& Bloemen 2011) with widths of 0.07 (roughly matching the level of agreement between models and observations, Müller et al. 2013). We explored parameter space with 100 walkers, which we evolved for 10000 steps each, discarding the first half for burn-in.

\subsection{Orbital Period}

Because we only observed a single transit of the planet candidate HD $283869 \mathrm{~b}$, the candidate's orbital period is not well determined. We therefore estimated the orbital period of HD $283869 \mathrm{~b}$ using a simplified version of the method described by Vanderburg et al. (2016a). We began by taking the posterior samples from our MCMC analysis of the $K 2$ light curve described in Section 3.4, which include 500,000 individual samples of the parameters $\left\{R_{p} / R_{\star}, t_{14}, b\right\}$. To estimate the orbital period of the planet, we took each set of these parameters drawn from the posterior, randomly drew samples of the eccentricity $e$ and argument of periastron $\omega$ from the joint distribution described by Kipping (2013, 2014), and calculated the orbital period $P$ by evaluating the following equation: $^{21}$

$$
P=\left[\frac{t_{14}\left(G M_{\star} \pi / 4\right)^{1 / 3}}{\sqrt{\left(R_{p}+R_{\star}\right)^{2}-b^{2} R_{\star}^{2}}} \frac{1+e \cos (\omega)}{\sqrt{1-e^{2}}}\right]^{3}
$$

where $G$ is the gravitational constant, $M_{\star}$ is the stellar mass, $R_{p}$ is the planetary radius, and $R_{\star}$ is the stellar radius. The resulting distribution of possible orbital periods for HD $283869 \mathrm{~b}$ peaks at about 40 days, with long tails extending to short periods inside of 10 days and long periods well beyond one year.

The duration, impact parameter, and planet-star radius ratio are not the only information we have at our disposal about the orbital period of HD 283869 b. We can also place constraints

\footnotetext{
21 This equation can be derived by simplifying Equation (2) from Vanderburg et al. (2016a) if the scaled semimajor axis $a / R_{\star} \gg 1$, a safe assumption for long-period transiting planet candidates like HD $283869 \mathrm{~b}$.
}

based on the fact that the planet candidate only transited once during the 80 days of $K 2$ observations. In particular, because the single transit occurred just about 8 days after the beginning of the $K 2$ observations, and no other similar dips occurred during the rest of the observing campaign, ${ }^{22}$ the candidate's orbital period must be longer than about 72 days. We accounted for this by discarding all samples of the transit parameters and orbital periods with periods less than this minimum allowed period.

We also took into account the probability that we would detect the transit of a long-period planet at all in our observations. When the orbital period of a planet is longer than the duration of observations, there is no guarantee that the transit will take place while observations are taking place. For orbital periods longer than the duration of observations $B$, the probability $\mathcal{P}$ of detecting a transit decreases as

$$
\mathcal{P}=\left(B+t_{14}\right) / P \text { for } P>B+t_{14} .
$$

We took this additional prior into account by randomly selecting whether to discard individual samples for periods longer than the observing baseline with a probability described by Equation (2).

We use the surviving samples to estimate both orbital and transit parameters for HD $283869 \mathrm{~b}$. The parameters are summarized in Table 1 and the orbital period probability distribution is shown in Figure 8. Most likely, the orbital period is not much longer than the minimum allowed period of 72 days; our analysis yields $P=106_{-25}^{+74}$ days. $^{23}$ Interestingly, given the luminosity and temperature of HD 283869, there is a fairly high likelihood that HD 283869 b orbits in the host star's habitable zone. $71 \%$ of the surviving orbital period samples fall within the optimistic habitable zone as calculated by Kopparapu et al. (2013), and $36 \%$ of the surviving samples fall within the conservative habitable zone. The equilibrium temperature of HD $283869 \mathrm{~b}$ is about $255_{-44}^{+38} \mathrm{~K}$, which would make it the first temperate planet found in an open cluster.

\section{Discussion}

\subsection{Uniqueness of HD $283869 \mathrm{~b}$}

If confirmed to be real, HD $283869 \mathrm{~b}$ would stand out among transiting planets in open clusters. With a $K$-band magnitude $K=7.7$, HD 283869 would be the brightest star to host a transiting planet in a cluster, making detailed further studies possible. The brightness and slow rotation of HD 283869 make it well suited for precise RV observations (though a detection of HD $283869 \mathrm{~b}$ may have to wait for advances in the treatment of stellar activity, see Section 4.3), and the brightness in the infrared and the fairly small size of the host star could make future transit transmission spectroscopy observations possible.

What sets HD $283869 \mathrm{~b}$ apart from the population of transiting planets in clusters is its long orbital period and low-irradiation environment. The longest-period validated transiting planet in a cluster is K2-136 d (Mann et al. 2018), which, with a period of 25.6 days, is the outermost planet in a

\footnotetext{
22 While Kepler observations during Campaign 13 were uninterrupted, our default light curve reduction excluded data from several short periods of time when the spacecraft briefly lost fine-pointing control. We re-reduced the $K 2$ light curve while including these data and confirmed that no transits occurred during these gaps (see Figure 7).

23 The orbital period is not particularly sensitive to the choice of eccentricity prior. If we assume the planet's orbit is circular, we find $P=99_{-20}^{+50}$ days.
} 

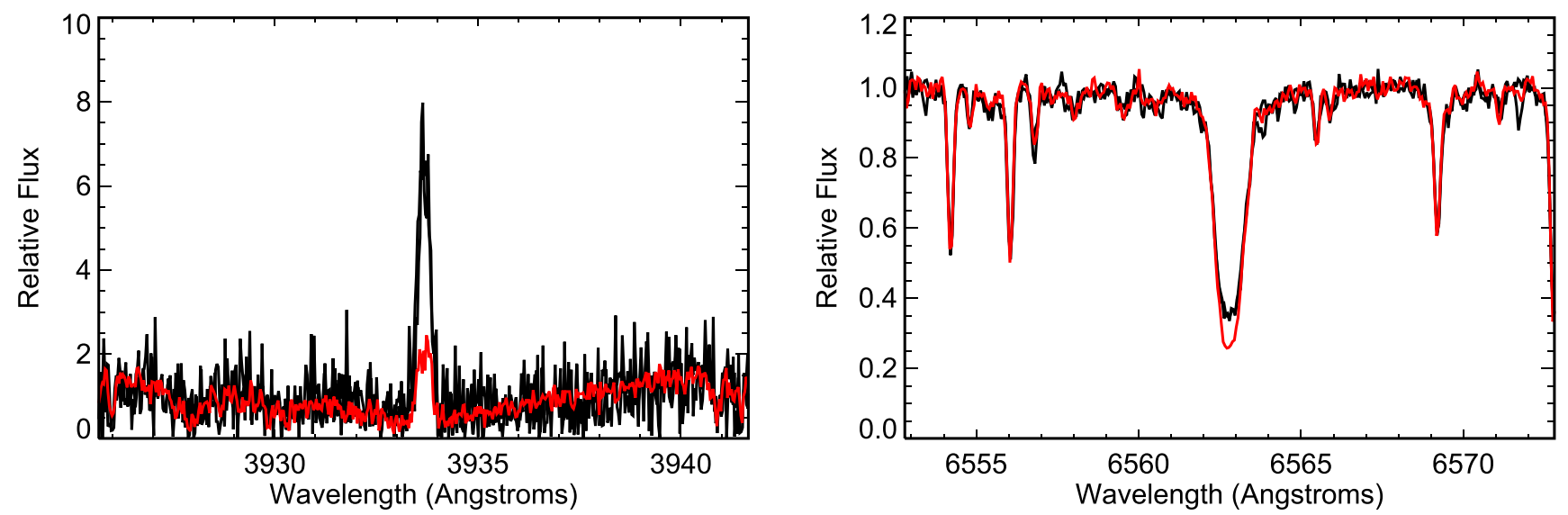

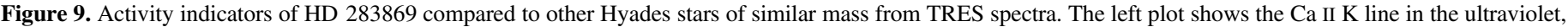

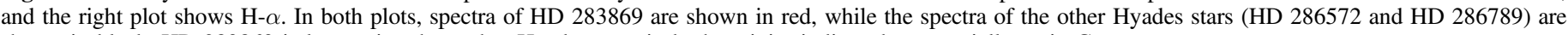
shown in black. HD 283869 is less active than other Hyades stars in both activity indices, but especially so in Ca II.

three-planet system. HD 283869 b likely has an orbital period more than three times longer than K2-136 d. HD $283869 \mathrm{~b}$ would also be the transiting cluster planet that receives the least stellar irradiation. HD $283869 \mathrm{~b}$ receives $1.2_{-0.6}^{+0.5}$ times the flux received by the Earth, four times less flux than is received by K2-103, the present record holder.

The combination of its young age, proximity, and lowirradiation make HD $283869 \mathrm{~b}$ an intriguing target for studying the development of small, temperate planets. At an age of roughly 600-800 million years, HD 283869 b may still be evolving into its mature state. Radius evolution models calculated by Lopez \& Fortney (2014) for super-Earths with hydrogen-rich envelopes predict that in the absence of photoevaporation, if HD $283869 \mathrm{~b}$ has a hydrogen-rich envelope, its radius will contract somewhere between $5 \%$ and $10 \%$ between now and maturity at an age of about 5 Gyr. Comparisons of the density of HD 283869 b to similar planets around older field stars could test these models. Observations of HD 283869 b might otherwise reveal surprises; other transiting planets discovered in the Hyades and Praesepe like K2-25 b and K2-95 b seem to be larger than their counterparts around mature stars (Mann et al. 2016a, 2017; Obermeier et al. 2016), indicating that processes like atmospheric evaporation may still be taking place. If transit observations of HD $283869 \mathrm{~b}$ show evidence for atmospheric loss, HD $283869 \mathrm{~b}$ might be the progenitor of an even smaller temperate planet, and potentially an early version of a rocky habitable-zone planet.

\subsection{Evidently Slow Rotation}

In Section 2.1, we identified a possible 37-day rotation period for HD 283869, which is considerably longer than the rotation periods of stars of similar mass and age in the Hyades and the similarly aged Praesepe open cluster. At face value, this is surprising. Several groups (Douglas et al. 2016, 2017; Rebull et al. 2017) have used $K 2$ data to measure rotation periods of Hyades and Praesepe stars and found tight period-mass relations for single stars in these clusters, with high $(\approx 85 \%)$ recovery fractions. A few other Hyades-age stars show longerperiod variability than their peers, including the Praesepe member EPIC 211974724 with a 35-day period (Agüeros et al. 2011; Douglas et al. 2017), but it is unclear whether these long rotation periods are actually reliable. HD 283869 also appears unusually inactive in spectroscopic indicators. For HD 283869, Mt. Wilson $R_{\mathrm{HK}}^{\prime}=-4.77$, while the median $R_{\mathrm{HK}}^{\prime}$ for Hyades stars is -4.47 with a scatter of 0.09 (Mamajek \& Hillenbrand 2008). We compare the Ca II K line for HD 283869 with those of similar Hyades stars in Figure 9. While HD 283869's H- $\alpha$ equivalent width is not easily distinguished from other Hyades-age stars in low-resolution spectra obtained by Douglas et al. (2014), inspection of high-resolution spectra of some of these stars shows HD 283869 is less active in H- $\alpha$ as well (see Figure 9).

One possibility for explaining the longer-period variability on HD 283869 and others like EPIC 211974724 is that we view these stars nearly pole-on and the variability timescale is dominated by the spot evolution timescale/activity lifetime rather than the stellar rotation period. This interpretation is consistent with our upper limit on the projected rotational velocity of about $2 \mathrm{~km} \mathrm{~s}^{-1}$. Interestingly, if true, this explanation would imply that the planet candidate, HD 283869 b, has an orbit significantly misaligned from its host's spin axis. A pole-on viewing geometry could also potentially explain the lower spectroscopic activity indicators as well if fewer active regions are visible from our line of sight.

Another more mundane possibility is that the long-period variability is instrumental in origin, and the true activity signal of HD 283869 is undetectable in the presence of long-timescale instrumental systematics. We think this explanation is unlikely. While Kepler and $K 2$ data do exhibit long-term systematics due to differential velocity aberration, the morphology of the longterm signal in the HD 283869 light curve does not match typical instrumental signals in $K 2$ data. If the signal were instrumental, its amplitude would be unusually high for a star of this brightness. Additionally, the amplitude and morphology of the signal does not depend on the size or shape of the photometric aperture used to extract the light curve. The longperiod signal is large enough that it should be detectable in ground-based observations that could clarify its origin. ${ }^{24}$

\subsection{Recovering and Confirming the Planet Candidate}

Confirming HD $283869 \mathrm{~b}$ and determining its orbital period with RV follow-up will be quite challenging. We estimate a planet mass of about $6.5 \pm 2 M_{\oplus}$ using the probabilistic massradius relationship from Wolfgang et al. (2016), which

\footnotetext{
24 The 35-day period detected on the Praesepe star EPIC 211974724 has already passed this test; the signal was detected both in $K 2$ and ground-based observations separated by 5 years, effectively ruling out instrumental artifacts (Agüeros et al. 2011; Douglas et al. 2017).
} 
corresponds to an RV semiamplitude of about $1.0 \pm 0.4 \mathrm{~m} \mathrm{~s}^{-1}$. While some short-period ${ }^{25}$ exoplanets with RV semiamplitudes this small have been detected, such small signals push against the limits of existing instrumentation and analysis techniques. Detecting such a small RV semiamplitude in the presence of the high-amplitude stellar activity signals expected for Hyadesage stars will be very difficult. Even in the optimistic case that HD 283869 has an unusually slow rotation period of 37 days, given the amplitude of photometric variations observed during the $K 2$ observations, we estimate the stellar activity would induce up to 6-8 $\mathrm{m} \mathrm{s}^{-1}$ peak-to-peak RV variations. Detecting the smaller signal of HD 283869 b in radial velocities may not be possible until instrumentation and analysis techniques have advanced.

The most straightforward path to confirming the transit signal and precisely measuring the orbital period of HD $283869 \mathrm{~b}$ is photometric monitoring to detect additional transits. The candidate's long orbital period and shallow depth make it infeasible to detect from the ground, so space-based monitoring is required. NASA's recently launched Transiting Exoplanet Survey Satellite (TESS) mission (Ricker et al. 2015) will not observe HD 283869 during its two-year prime mission because it lies too close to the ecliptic plane, but it could observe HD 283869 in an extended mission. In particular, some of the extended mission concepts proposed by Bouma et al. (2017) observe the ecliptic plane for periods of time ranging from 14 days to up to 112 days. If one of these longer ecliptic pointings were to be adopted as a TESS extended mission, it could detect a transit of HD 283869 b. The orbital period of the planet is probably just a bit longer than the 72-day minimum allowed orbital period, and TESS should be able to detect a transit of HD 283869 b with a signal-to-noise ratio of about 11 (Jaffe \& Barclay 2017; Stassun et al. 2017). The confirmation of a habitable-zone super-Earth in an open cluster would be a strong example of how K2-TESS synergy can strengthen the legacy of both missions.

We thank Luke Bouma for helpful discussions about TESS extended mission strategies, and we thank the anonymous referee for a helpful and constructive review. This work was performed in part under contract with the California Institute of Technology/Jet Propulsion Laboratory funded by NASA through the Sagan Fellowship Program executed by the NASA Exoplanet Science Institute. A.W.M. was supported through Hubble Fellowship grant 51364 awarded by the Space Telescope Science Institute, which is operated by the Association of Universities for Research in Astronomy, Inc., for NASA, under contract NAS 5-26555. A.C.R. was supported (in part) by NASA $K 2$ Guest Observer Cycle 4 grant NNX17AF71G. D.W.L. acknowledges partial support from the TESS mission through a sub-award from the Massachusetts Institute of Technology to the Smithsonian Astrophysical Observatory (SAO) and from the Kepler mission under NASA Cooperative agreement NNX13AB58A with SAO.

This research has made use of NASA's Astrophysics Data System and the NASA Exoplanet Archive, which is operated by the California Institute of Technology, under contract with the National Aeronautics and Space Administration under the

\footnotetext{
${ }^{25}$ The long orbital period of HD 283869 poses an additional challenge. Most advances in treating stellar activity signals have been for exoplanets with orbital periods shorter than the stellar rotation period (e.g., Haywood et al 2014)
}

Exoplanet Exploration Program. The National Geographic Society-Palomar Observatory Sky Atlas (POSS-I) was made by the California Institute of Technology with grants from the National Geographic Society. The Oschin Schmidt Telescope is operated by the California Institute of Technology and Palomar Observatory.

This paper includes data collected by the Kepler mission. Funding for the Kepler mission is provided by the NASA Science Mission directorate. Some of the data presented in this paper were obtained from the Mikulski Archive for Space Telescopes (MAST). STScI is operated by the Association of Universities for Research in Astronomy, Inc., under NASA contract NAS5-26555. Support for MAST for non-HST data is provided by the NASA Office of Space Science via grant NNX13AC07G and by other grants and contracts. This work has made use of data from the European Space Agency (ESA) mission Gaia (https:// www.cosmos.esa.int/gaia), processed by the Gaia Data Processing and Analysis Consortium (DPAC, https://www.cosmos.esa. int/web/gaia/dpac/consortium). Funding for the DPAC has been provided by national institutions, in particular the institutions participating in the Gaia Multilateral Agreement.

Some observations in the paper made use of the NNEXPLORE Exoplanet and Stellar Speckle Imager (NESSI). NESSI was funded by the NASA Exoplanet Exploration Program and the NASA Ames Research Center. NESSI was built at the Ames Research Center by Steve B. Howell, Nic Scott, Elliott P. Horch, and Emmett Quigley. The NESSI data were obtained at the WIYN Observatory from telescope time allocated to NN-EXPLORE through the scientific partnership of the National Aeronautics and Space Administration, the National Science Foundation, and the National Optical Astronomy Observatory.

We wish to recognize and acknowledge the very significant cultural role and reverence that the summit of Maunakea has always had within the indigenous Hawaiian community. We are most fortunate to have the opportunity to conduct observations from this mountain. We are also honored to be permitted to conduct observations on Iolkam Du'ag (Kitt Peak), a mountain within the Tohono O'odham Nation with particular significance to the Tohono O'odham people.

Facilities: Kepler/K2, FLWO:1.5 m (TRES, CfA Digital Speedometers), WIYN (NESSI), Gemini:Gillett ('Alopeke), ORO:Wyeth (CfA Digital Speedometers), Gaia, Exoplanet Archive, MAST, CDS, ADS.

\section{ORCID iDs}

Andrew Vanderburg (iD https://orcid.org/0000-0001-7246-5438 Andrew W. Mann (1) https://orcid.org/0000-0003-3654-1602 Aaron Rizzuto (i) https://orcid.org/0000-0001-9982-1332 Allyson Bieryla (iD https://orcid.org/0000-0001-6637-5401 Adam L. Kraus (10 https://orcid.org/0000-0001-9811-568X Michael L. Calkins (iD https://orcid.org/0000-0002-28305661

Jason L. Curtis (1) https://orcid.org/0000-0002-2792-134X Stephanie T. Douglas (i) https://orcid.org/0000-00017371-2832

Mark E. Everett (10) https://orcid.org/0000-0002-0885-7215

Elliott P. Horch (iD https://orcid.org/0000-0003-2159-1463

Steve B. Howell (i) https://orcid.org/0000-0002-2532-2853

David W. Latham (iD https://orcid.org/0000-0001-9911-7388

Samuel N. Quinn (1D https://orcid.org/0000-0002-8964-8377

Nicholas J. Scott (iD https://orcid.org/0000-0003-1038-9702 


\section{References}

Agüeros, M. A., Covey, K. R., Lemonias, J. J., et al. 2011, ApJ, 740, 110 Allard, F., Homeier, D., \& Freytag, B. 2011, in ASP Conf. Ser. 448, 16th Cambridge Workshop on Cool Stars, Stellar Systems, and the Sun, ed. C. Johns-Krull, M. K. Browning, \& A. A. West (San Francisco, CA: ASP), 91

Altmann, M., Roeser, S., Demleitner, M., Bastian, U., \& Schilbach, E. 2017, A\&A, 600, L4

Barnes, S. A. 2007, ApJ, 669, 1167

Bessell, M., \& Murphy, S. 2012, PASP, 124, 140

Boss, A. P. 1995, Sci, 267, 360

Bouma, L. G., Winn, J. N., Kosiarek, J., \& McCullough, P. R. 2017, arXiv: 1705.08891

Brandt, T. D., \& Huang, C. X. 2015, ApJ, 807, 24

Buchhave, L. A., Bizzarro, M., Latham, D. W., et al. 2014, Natur, 509, 593

Buchhave, L. A., Bakos, G. Á, Hartman, J. D., et al. 2010, ApJ, 720, 1118

Buchhave, L. A., Latham, D. W., Johansen, A., et al. 2012, Natur, 486, 375

Burke, C. J., Gaudi, B. S., DePoy, D. L., \& Pogge, R. W. 2006, AJ, 132, 210

Butler, R. P., Marcy, G. W., Williams, E., Hauser, H., \& Shirts, P. 1997, ApJL, 474, L115

Campbell, B., \& Walker, G. A. H. 1979, PASP, 91, 540

Campbell, B., Walker, G. A. H., \& Yang, S. 1988, ApJ, 331, 902

Charbonneau, D., Berta, Z. K., Irwin, J., et al. 2009, Natur, 462, 891

Choi, J., Dotter, A., Conroy, C., et al. 2016, ApJ, 823, 102

Ciardi, D. R., Crossfield, I. J. M., Feinstein, A. D., et al. 2018, AJ, 155, 10

Claret, A., \& Bloemen, S. 2011, A\&A, 529, A75

Cochran, W. D., Hatzes, A. P., Butler, R. P., \& Marcy, G. W. 1997, ApJ, 483,457

Cochran, W. D., Hatzes, A. P., \& Paulson, D. B. 2002, AJ, 124, 565

Cohen, M., Wheaton, W. A., \& Megeath, S. T. 2003, AJ, 126, 1090

Curtis, J. L., Vanderburg, A., Torres, G., et al. 2018, AJ, 155, 173

Cushing, M. C., Rayner, J. T., \& Vacca, W. D. 2005, ApJ, 623, 1115

David, T. J., Conroy, K. E., Hillenbrand, L. A., et al. 2016a, AJ, 151, 112

David, T. J., Hillenbrand, L. A., Petigura, E. A., et al. 2016b, Natur, 534, 658

David, T. J., Mamajek, E. E., Vanderburg, A., et al. 2018, arXiv:1801.07320

Dawson, R. I., Murray-Clay, R. A., \& Johnson, J. A. 2015, ApJ, 798, 66

Dotter, A. 2016, ApJS, 222, 8

Dotter, D., Chaboyer, A., Jevremović, B., et al. 2008, ApJS, 178, 89

Douglas, S. T., Agüeros, M. A., Covey, K. R., et al. 2014, ApJ, 795, 161

Douglas, S. T., Agüeros, M. A., Covey, K. R., et al. 2016, ApJ, 822, 47

Douglas, S. T., Agüeros, M. A., Covey, K. R., \& Kraus, A. 2017, ApJ, 842, 83

Dutra-Ferreira, L., Pasquini, L., Smiljanic, R., Porto de Mello, G. F., \& Steffen, M. 2016, A\&A, 585, A75

ESA 1997, ESA Special Publication, Vol. 1200, The HIPPARCOS and TYCHO Catalogues. Astrometric and Photometric Star Catalogues Derived from the ESA HIPPARCOS Space Astrometry Mission (Noordwijk: ESA), 1200

Gaia Collaboration, Brown, A. G. A., Vallenari, A., et al. 2018, arXiv:1804. 09365

Gaia Collaboration, Brown, A. G. A., Vallenari, A., et al. 2016a, A\&A, 595, A2

Gaia Collaboration, Prusti, T., de Bruijne, J. H. J., et al. 2016b, A\&A, 595, A1

Gaidos, E., Mann, A. W., Lépine, S., et al. 2014, MNRAS, 443, 2561

Geller, A. M., \& Mathieu, R. D. 2011, Natur, 478, 356

Gilliland, R. L., Brown, T. M., Guhathakurta, P., et al. 2000, ApJL, 545, L47

Goodman, J., \& Weare, J. 2010, Communications in Applied Mathematics and Computational Science, 5, 65

Griffin, R. F., Griffin, R. E. M., Gunn, J. E., \& Zimmerman, B. A. 1988, AJ, 96, 172

Haywood, R. D., Collier Cameron, A., Queloz, D., et al. 2014, MNRAS, 443,2517

Henden, A. A., Levine, S. E., Terrell, D., Smith, T. C., \& Welch, D. 2012, JAAVSO, 40, 430

Henry, T. J., \& McCarthy, D. W., Jr. 1993, AJ, 106, 773

Høg, E., Fabricius, C., Makarov, V. V., et al. 2000, A\&A, 355, L27

Howell, S. B., Everett, M. E., Sherry, W., Horch, E., \& Ciardi, D. R. 2011, AJ, 142,19

Howell, S. B., Sobeck, C., Haas, M., et al. 2014, PASP, 126, 398

Huber, D., Bryson, S. T., Haas, M. R., et al. 2016, ApJS, 224, 2

Jaffe, T. J., \& Barclay, T. 2017, Ticgen: A Tool for Calculating a TESS Magnitude, and an Expected Noise Level for Stars to be Observed by TESS, Zenodo, doi: $10.5281 /$ zenodo. 888217

Kipping, D. M. 2013, MNRAS, 434, L51

Kipping, D. M. 2014, MNRAS, 444, 2263
Kopparapu, R. K., Ramirez, R., Kasting, J. F., et al. 2013, ApJ, 765, 131

Kovács, G., Zucker, S., \& Mazeh, T. 2002, A\&A, 391, 369

Lallement, R., Welsh, B. Y., Vergely, J. L., Crifo, F., \& Sfeir, D. 2003, A\&A, 411,447

Latham, D. W., Stefanik, R. P., Mazeh, T., Mayor, M., \& Burki, G. 1989, Natur, 339, 38

Leiner, E., Mathieu, R. D., Stello, D., Vanderburg, A., \& Sandquist, E. 2016, ApJL, 832, L13

Libralato, M., Nardiello, D., Bedin, L. R., et al. 2016, MNRAS, 463, 1780

Liu, F., Yong, D., Asplund, M., Ramírez, I., \& Meléndez, J. 2016, MNRAS, 457, 3934

Livingston, J. H., Dai, F., Hirano, T., et al. 2018, AJ, 155, 115

Lopez, E. D., \& Fortney, J. J. 2014, ApJ, 792, 1

Lovis, C., \& Mayor, M. 2007, A\&A, 472, 657

Mamajek, E. E., \& Hillenbrand, L. A. 2008, ApJ, 687, 1264

Mandel, K., \& Agol, E. 2002, ApJL, 580, L171

Mann, A. W., Feiden, G. A., Gaidos, E., Boyajian, T., \& von Braun, K. 2015 , ApJ, 804, 64

Mann, A. W., Gaidos, E., Mace, G. N., et al. 2016a, ApJ, 818, 46

Mann, A. W., Gaidos, E., Vanderburg, A., et al. 2017, AJ, 153, 64

Mann, A. W., Newton, E. R., Rizzuto, A. C., et al. 2016b, AJ, 152, 61

Mann, A. W., Vanderburg, A., Rizzuto, A. C., et al. 2018, AJ, 155, 4

Mann, A. W., \& von Braun, K. 2015, PASP, 127, 102

Martín, E. L., Lodieu, N., Pavlenko, Y., \& Béjar, V. J. S. 2018, ApJ, 856, 40

Masuda, K., \& Winn, J. N. 2017, AJ, 153, 187

Masuda, K. 2014, ApJ, 783, 53

Mayo, A. W., Vanderburg, A., Latham, D. W., et al. 2018, arXiv:1802.05277

Mayor, M., \& Queloz, D. 1995, Natur, 378, 355

Meibom, S., Torres, G., Fressin, F., et al. 2013, Natur, 499, 55

Mermilliod, J.-C., Mayor, M., \& Udry, S. 2009, A\&A, 498, 949

Mermilliod, J.-C., Mermilliod, M., \& Hauck, B. 1997, A\&AS, 124, 349

Morton, T. D., Bryson, S. T., Coughlin, J. L., et al. 2016, ApJ, 822, 86

Muiños, J. L., \& Evans, D. W. 2014, AN, 335, 367

Müller, H. M., Huber, K. F., Czesla, S., Wolter, U., \& Schmitt, J. H. M. M. 2013, A\&A, 560, A112

Naef, D., Latham, D. W., Mayor, M., et al. 2001, A\&A, 375, L27

Obermeier, C., Henning, T., Schlieder, J. E., et al. 2016, AJ, 152, 223

Paulson, D. B., Cochran, W. D., \& Hatzes, A. P. 2004, AJ, 127, 3579

Paulson, D. B., Sneden, C., \& Cochran, W. D. 2003, AJ, 125, 3185

Pepper, J., Stanek, K. Z., Pogge, R. W., et al. 2008, AJ, 135, 907

Perryman, M. A. C., Brown, A. G. A., Lebreton, Y., et al. 1998, A\&A, 331, 81

Quinn, S. N., White, R. J., Latham, D. W., et al. 2012, ApJL, 756, L33

Rayner, J. T., Cushing, M. C., \& Vacca, W. D. 2009, ApJS, 185, 289

Rebull, L. M., Stauffer, J. R., Hillenbrand, L. A., et al. 2017, ApJ, 839, 92

Ricker, G. R., Winn, J. N., Vanderspek, R., et al. 2015, JATIS, 1, 014003

Rizzuto, A. C., Ireland, M. J., \& Kraus, A. L. 2015, MNRAS, 448, 2737

Rizzuto, A. C., Ireland, M. J., \& Robertson, J. G. 2011, MNRAS, 416, 3108

Rizzuto, A. C., Mann, A. W., Vanderburg, A., Kraus, A. L., \& Covey, K. R. 2017, AJ, 154, 224

Rogers, L. A. 2015, ApJ, 801, 41

Röser, S., Schilbach, E., Piskunov, A. E., Kharchenko, N. V., \& Scholz, R.-D. 2011, A\&A, 531, A92

Rowe, J. F., Bryson, S. T., Marcy, G. W., et al. 2014, ApJ, 784, 45

Russell, H. N. 1914, PA, 22, 275

Sato, B., Izumiura, H., Toyota, E., et al. 2007, ApJ, 661, 527

Shapley, H. 1917, ApJ, 45, 118

Skrutskie, M. F., Cutri, R. M., Stiening, R., et al. 2006, AJ, 131, 1163

Stassun, K. G., Oelkers, R. J., Pepper, J., et al. 2017, arXiv:1706.00495

Stefanik, R. P., \& Latham, D. W. 1985, in IAU Coll. 88, Stellar Radial Velocities, ed. A. G. D. Philip \& D. W. Latham (Schenectady, NY: L. Davis Press), 213

Stern, R. A., Zolcinski, M. C., Antiochos, S. K., \& Underwood, J. H. 1981, ApJ, 249, 647

Struve, O. 1952, Obs, 72, 199

van Cleve, J. E., \& Caldwell, D. A. 2016, Kepler Instrument Handbook, Tech. rep. KSCI-19033-002

van Leeuwen, F., Evans, D. W., Grenon, M., et al. 1997, A\&A, 323, L61

van Saders, J. L., \& Gaudi, B. S. 2011, ApJ, 729, 63

Vanderburg, A. 2014, arXiv:1412.1827

Vanderburg, A., Becker, J. C., Kristiansen, M. H., et al. 2016a, ApJL, 827, L10

Vanderburg, A., \& Johnson, J. A. 2014, PASP, 126, 948

Vanderburg, A., Latham, D. W., Buchhave, L. A., et al. 2016b, ApJS, 222, 14

Vanderburg, A., Montet, B. T., Johnson, J. A., et al. 2015, ApJ, 800, 59

Wolfgang, A., Rogers, L. A., \& Ford, E. B. 2016, ApJ, 825, 19

Wright, E. L., Eisenhardt, P. R. M., Mainzer, A. K., et al. 2010, AJ, 140, 1868 